Public Abstract

First Name:Daniel

Middle Name:Paul

Last Name:Caputo

Adviser's First Name:Angela

Adviser's Last Name:Speck

Co-Adviser's First Name:

Co-Adviser's Last Name:

Graduation Term:SP 2010

Department:Physics

Degree:MS

Title:Investigating the Formation Mechanism of Polycyclic Aromatic Hydrocarbons and

Adapting Particle Swarm Optimization Techniques to Search Large Data Sets

\title{
Polycyclic Aromatic Hydrocarbons
}

The unidentified infrared bands (UIBs) have been attributed to emission from polycyclic aromatic hydrocarbons (PAHs). The UIBs at 3.3 and $11.3 \hat{1} 1 / 4 \mathrm{~m}$, among others, are seen in many astrophysical environments, with the notable exception of carbon-rich AGB stars (C stars). PAHs are expected to form around $\mathrm{C}$ stars, yet only a few show the UIBs and all of these have hot companions. This makes $\mathrm{C}$ stars with hot companions an ideal environment to study the conditions associated with PAH formation and processing.

The precise wavelengths and ratios of the features depend on the size, composition, and charge state of the individual PAHs. Additionally, the wavelength of photons needed to excite PAHs depends on the individual PAHs size and charge state. While small PAHs undoubtedly need higher energy (UV) photons, it has been suggested that large ( $>50 \mathrm{C}$ atoms) or ionized PAHS can be excited by visible or even near-IR photons. The lack of PAH emission from single carbon stars suggests that either PAHs do not form around C stars, or that only small neutral grains form which cannot be excited by a C starâ€ ${ }^{T M}$ s soft radiation field.

We present new observations of several $\mathrm{C}$ stars with hot companions in order to understand the distribution of PAHs responsible for the emerging UIB emission. By comparing the distributions of these emissions, we attempt to build a coherent picture of the size and charge state of PAHs around these carbon stars, and how that varies by location. This will provide the basis for understanding the formation and processing of PAHs.

\section{Particle Swarm Optimization}

As the size of data and model sets continue to increase, more efficient ways are needed to sift through the available information. We present a computational method which will efficiently search large parameter spaces to either map the space or find individual data/models of interest.

Particle swarm optimization (PSO) is a subclass of artificial life computer algorithms. The PSO algorithm attempts to leverage â€œswarm intelligenceâ€• against finding optimal solutions to a problem. This system is ofte based on a biological model of a swarm (e.g. schooling fish). These biological models are broken down into a few simple rules which govern the behavior of the system. â€œAgentsâ€• (e.g. fish) are introduced and the agents, following the rules, search out solutions much like a fish would seek out food. We have made extensive modifications to the standard PSO model which increase its efficiency as-well-as adding the capacity to map a parameter space and find multiple solutions. 